\title{
$1 \quad$ Zika virus NS3 protease and its cellular substrates
}

2

3 Agnieszka Dabrowska ${ }^{\mathrm{a}, \mathrm{b}}$, Aleksandra Milewska ${ }^{\mathrm{a}, \mathrm{b}}$, Joanna Ner-Kluza ${ }^{\mathrm{c}}$, Piotr Suder , 4 Krzysztof Pyrc ${ }^{\mathrm{a}, *}$

5

$6 \quad{ }^{a}$ Virogenetics Laboratory of Virology, Malopolska Centre of Biotechnology, Jagiellonian 7 University, Gronostajowa 7a, 30-387 Krakow, Poland.

8 b Microbiology Department, Faculty of Biochemistry, Biophysics and Biotechnology, 9 Jagiellonian University, Gronostajowa 7, 30-387 Krakow, Poland.

$10{ }^{\mathrm{c}}$ Department of Biochemistry and Neurobiology, Faculty of Materials Science and Ceramics, 11 AGH University of Science and Technology, Mickiewicza 30, 30-059 Krakow, Poland.

$13{ }^{*}$ Corresponding author

14

$15 *$ Correspondence should be addressed to Krzysztof Pyrc, Virogenetics Laboratory of 16 Virology, Malopolska Centre of Biotechnology, Jagiellonian University, Gronostajowa 7a, 17 30-387 Krakow, Poland; Phone number: +48 1266461 21; www: http://virogenetics.info, E18 mail: k.a.pyrc@uj.edu.pl 


\section{ABSTRACT}

21 Zika virus is a flavivirus discovered in 1947, but the association between Zika virus

22 infection and brain disorders was not demonstrated until 2015 in Brazil. Infection mostly poses

23 a threat to women during pregnancy, since it may cause microcephaly and other neurological

24 dysfunctions in the developing fetus. However, infection is also associated with Guillain-Barré

25 syndrome. The nonstructural NS3 protein is essential for virus replication because it helps to

26 remodel the cellular microenvironment. Several reports show that this protease can process

27 cellular substrates and thereby modify cellular pathways that are important for the virus. Herein,

28 we explored some of the targets of NS3, but we could not confirm the biological relevance of

29 its protease activity. Thus, although mass spectrometry is highly sensitive and useful in many

30 instances, being also able to show directions, where cell/virus interaction occurs, we believe

31 that biological validation of the observed results is essential. 


\section{INTRODUCTION}

Zika virus is a flavivirus discovered in 1947 in primates inhabiting the African Zika forest (1). Although the virus was found to infect humans, for decades it was not considered to be a medical threat due to limited distribution and very mild symptoms associated with infection. However, more than 10 years ago interest in Zika virus began to increase as it became clear that the virus has broadened its geographic distribution, and the first outbreak was reported in the Federated States of Micronesia (2). In 2015, case definition became more precise, and some data suggested that the infection may be more dangerous than previously thought. While the symptoms are relatively mild and include fever, rash, headache, and muscle pain, infection may cause severe sequelae, and it is associated with Guillain-Barré syndrome (3). The infection is most severe in pregnant women, since it interferes with development of the neurological system of the fetus, predominantly resulting in microcephaly. A number of in vitro and in vivo studies have confirmed these observations (4-10).

Flaviviruses are small, enveloped viruses with positive-strand RNA genome, which is delivered to the target cell as a single-stranded RNA molecule containing a single open reading frame (ORF). This ORF is translated into an immature polyprotein, which is co- and posttranslationally cleaved by viral and cellular proteases to yield 10 mature viral proteins; capsid (C), membrane (prM/M), and envelope (E) structural proteins; and seven nonstructural proteins (NS1, NS2A, NS2B, NS3, NS4A, NS4B, and NS5)(11). Cleavage sites processed by the viral serine NS3 protease are located between NS2A/NS2B, NS2B/NS3, NS3/NS4A, and NS4B/NS5. Furin or similar cellular proteases process the prM/M site, while other host cell proteases reportedly cleave $\mathrm{C} / \mathrm{prM}$, prM/E, E/NS1, NS1/NS2A, and NS4A/NS4B sites(12). The NS3 protein consists of an N-terminal serine protease domain ( $\sim 180$ amino acids) and a C-terminal region harboring RNA helicase, nucleoside triphosphatase, and 5' RNA triphosphatase activities. The active site of the N-terminal protease contains a His-Asp-Ser 
57 catalytic triad. Furthermore, the small nonstructural NS2B protein serves as an NS3 protease

58 cofactor and anchors it to the endoplasmic reticulum (ER) membrane (13-17). The presence or

59 absence of NS2B affects the tertiary structure, activity, and stability of NS3 (18-20).

60 Flaviviral proteases are essential for viral replication, hence they are considered promising

61 targets for antiviral agents. Indeed, the development of HCV NS3/NS4A protease inhibitors

62 proved a breakthrough in hepatitis C therapy, and these drugs received U.S. Food \& Drug

63 Administration (FDA) and European Medicines Agency (EMA) approval for use in humans

$64(21-23)$.

65 Interestingly, flaviviral proteases have also been reported to modify the cellular

66 microenvironment. Cleavage of host proteins may be beneficial for the virus by diminishing

67 the cellular responses, remodeling cellular metabolism, and other mechanisms. Such a strategy

68 is common for viruses, as exemplified by human rhinovirus (HRV) that modulates apoptosis

69 by cleaving receptor-interacting protein kinase-1 (RIPK1) at the noncanonical site, and

70 blocking caspase 8-mediated activation of the pathway (24). Interestingly, the picornaviral

71 protease also processes translation initiation factor eIF4G, part of the cellular translation

72 initiation complex. Targeting of this molecule results in decreased production of cellular

73 proteins but does not affect the production of viral proteins, as picornaviruses use internal

74 ribosome entry sites (IRES) for cap-independent translation. In this way, the viral protease

75 hijacks the cellular protein production machinery (25-27). The NS3/NS4A protease of hepatitis

76 C virus cleaves mitochondrial antiviral signaling protein (MAVS) and TIR domain-containing

77 adapter-inducing interferon- $\beta$ protein (TRIF) to evade the host cell antiviral response (28-30).

78 Various targets of the Zika virus protease have been identified. FAM134B (family with

79 sequence similarity 134), ATG16L1 (autophagy-related protein 16-1), eIF4G1 (eukaryotic

80 translation initiation factor 4 gamma 1), and Septin-2 are among the most interesting targets.

81 Except for FAM134B, these targets were identified using mass spectrometry methods, which 
82 provide unrivalled sensitivity and are capable of cell proteome studies. However, careful

83 analysis of the reported data showed that none of the protein targets are shared between the

84 published studies. Thus, we explored the reported data in detail by employing classical

85 approaches such as western blotting, as well as functional approaches based on the activity of

86 particular pathways. Herein, we present an example of such a study, in which we failed to

87 confirm protease-mediated or virus-related degradation of the eIF4G1 protein. We were also

88 unable to confirm the beneficial effects of decreasing eIF4G1 on viral replication. 


\section{MATERIALS AND METhODS}

90

91

92

93

94

\section{Cell culture}

293 T cells (ATCC CRL-3216; human embryonic kidney cells), A549 cells (ATCC CCL185; lung carcinoma cells), Vero cells (ATCC CCL-81; African green monkey kidney cells), and U251 cells (human glioblastoma cell line) were maintained in Dulbecco's modified Eagle's medium (DMEM; Corning, Poland) supplemented with 3\% fetal bovine serum (FBS; heatinactivated; Thermo Scientific, Poland), $100 \mu \mathrm{g} / \mathrm{ml}$ streptomycin, $100 \mathrm{U} / \mathrm{ml}$ penicillin (SigmaAldrich, Poland), and $5 \mu \mathrm{g} / \mathrm{ml}$ ciprofloxacin. Cells were maintained at $37^{\circ} \mathrm{C}$ under $5 \% \mathrm{CO}_{2}$.

\section{Virus strains, preparation, and titration}

ZIKV H/PF/2013 (acquired from European Virus Archive), ZIKV H/PAN/2016 (BEI resources), ZIKV R116265 Human 2016 Mexico (BEI resources), ZIKV Mosquito Mex 2-81 (BEI resources), ZIKV PRVABC59 (BEI resources), ZIKV MR766 (BEI resources), ZIKV IB H 30656 (BEI resources), ZIKV FLR (BEI resources), ZIKV R103451 Human 2015 Honduras (BEI resources), ZIKV P 6-740 Malaysia 1966 (BEI resources), and ZIKV DAKAR 41524 (BEI resources) strains were employed in this work.

Virus stocks were generated by infection of Vero cells. At 3 days post-infection (p.i.) at $37^{\circ} \mathrm{C}$, virus-containing medium was collected and titrated. As a control, mock-infected Vero cells were subjected to the same procedure. Virus and mock aliquots were stored at $-80^{\circ} \mathrm{C}$. Virus titration was performed on confluent Vero cells in a 96-well plate according to the method described by Reed-Muench(31). Briefly, cells infected with serially diluted virus were incubated at $37^{\circ} \mathrm{C}$ for 3 days, and the occurrence of a cytopathic effect (CPE) was monitored. 


\section{Plasmids}

113 The region encoding the NS2B-NS3 ${ }^{\text {WT }}$ protein was amplified by PCR using a cDNA 114 template generated from H/FP/2013 Zika virus and appropriate primers (5' ATG CGG TAC 115 CGC CAC CAT GGG CAG CTG GCC CCC TAG CGA A 3’; 5’ AGC CGG TAC CCT ATC

116 TTT TCC CAG CGG CAA ACT CC 3'). The resulting product was digested with NotI-HF and

117 KpnI-HF (New England Biolabs), gel-purified, and cloned into the pBudCE4.1 vector 118 (pBudCE4.1_NS3 ${ }^{\mathrm{WT}}$ ). The plasmid encoding the inactive NS2B-NS3 ${ }^{\mathrm{S} 135 \mathrm{~A}}$ protease 119 (pBudCE4.1-NS3 ${ }^{\mathrm{S} 135 \mathrm{~A}}$ ) was obtained using the pBudCE4.1_NS3 ${ }^{\mathrm{WT}}$ template by employing the 120 QuickChange PCR technique with appropriate primers (5' GGA ACT GCC GGA TCT CCA ATC CTA GAC AAG 3'; 5’ AGA TCC GGC AGT TCC TGC TGG GTA ATC CAG 3') to

122 change the serine residue at amino acid (aa) position 135 to alanine. The obtained plasmids 123 were verified by DNA sequencing.

\section{Plasmid transfection}

126 293T cells were maintained as described above. Cells were seeded in 6- or 24-wells plates

127 (TPP, Switzerland) and cultured for $24 \mathrm{~h}$. When $60 \%$ confluency was reached, cells were 128 transfected using polyethyleneimine (PEI; Sigma-Aldrich, Poland). For transfection in 6-wells 129 plates, $4 \mu$ g plasmid DNA was mixed with $250 \mu$ l Opti-MEM medium (Thermo Scientific) and $1304 \mu \mathrm{g}$ PEI. For transfection in 24-well plates, $1 \mu \mathrm{g} /$ well plasmid DNA was mixed with $100 \mu \mathrm{l}$ 131 Opti-MEM medium with $1 \mu$ g PEI. After a 30 min incubation at room temperature, the mixture 132 was added dropwise onto cells. Four hours later, the supernatant was discarded, fresh medium 133 was added, and cells were further incubated at $37^{\circ} \mathrm{C}$.

134 A549 cells were maintained as described above. Cells were seeded in 6-wells plates and 135 cultured for $24 \mathrm{~h}$. When $80 \%$ confluency was reached, cells were transfected with 136 Lipofectamine 2000 (Thermo Scientific) according to the manufacturer's protocol. Briefly, 
$1372.5 \mu$ g plasmid DNA was mixed with $300 \mu$ l Opti-MEM medium with $5 \mu$ l Lipofectamine 2000.

138 After a 5 min incubation at room temperature, the mixture was added dropwise onto cells. Four

139 hours later, the supernatant was discarded, fresh medium was added, and cells were further 140 incubated at $37^{\circ} \mathrm{C}$.

141 For expression of active and inactive virus protease, pBudCE4.1-NS3 ${ }^{\mathrm{WT}}$ or pBudCE4.1-

$142 \mathrm{NS}^{\mathrm{S} 135 \mathrm{~A}}$ plasmids were employed, respectively. For eIF4G1 overexpression, cells were

143 transfected with pcDNA3 HA eIF4GI plasmid or control green fluorescent protein (GFP)-

144 expressing plasmid (pMAX-GFP plasmid, Lonza). pcDNA3 HA eIF4GI (1-1599) was a gift

145 from Nahum Sonenberg (Addgene plasmid \#45640; http://n2t.net/addgene:45640; RRID

146 Addgene_45640). The efficiency of expression was verified by western blotting.

siRNA transfection

149 For small interfering RNA (siRNA) transfection, A549 cells were maintained as described

150 above. Cells were seeded in 24-wells plates, and siRNA was transfected once the confluency

151 reached $80 \%$ using RNAiMAX Lipofectamine (Thermo Scientific), according to the

152 manufacturer's protocol. Next, 5 pmol eIF4G1 siRNA (Sigma-Aldrich; Cat. No EHU066831)

153 or control scrambled RNA (Santa-Cruz Biotechnology; Cat. No sc-44237) was mixed in $125 \mu 1$

154 Opti-MEM medium containing $3.5 \mu$ transfection reagent. After a 5 min incubation at room 155 temperature, the mixture was added to cells dropwise. The efficiency of eIF4G1 silencing was 156 verified at $24-72 \mathrm{~h}$ post-transfection using western blotting.

\section{Virus infection}

$159293 \mathrm{~T}$ cells, A549 cells, and U251 cells were seeded in 6-wells plates and cultured at $37^{\circ} \mathrm{C}$.

160 When $90-100 \%$ confluency was reached, cells were inoculated with ZIKV at $2000 \mathrm{TCID} 50 / \mathrm{ml}$ 161 for $293 \mathrm{~T}$ cells or $400 \mathrm{TCID}_{50} / \mathrm{ml}$ for U251 and A549 cells. Mock cultures were inoculated with 
162 an identical volume of mock samples. All cultures were incubated for $2 \mathrm{~h}$ at $37^{\circ} \mathrm{C}$ under $5 \%$

$163 \mathrm{CO}_{2}$ in DMEM medium supplemented with $2 \% \mathrm{FBS}, 100 \mu \mathrm{g} / \mathrm{ml}$ streptomycin, and $100 \mathrm{IU} / \mathrm{ml}$

164 penicillin. After incubation, cells were washed twice with phosphate-buffered saline (PBS) and

165 incubated as described above. At 3 days p.i., culture supernatants were collected, viral RNA

166 was isolated, and the yield was quantified by reverse transcription quantitative PCR (RT-

167 qPCR). Also, cells were collected for western blotting analysis.

168

169

SUnSET-puromycin assay

170 A549 and U251 cells were maintained as described above. Cells were seeded in 12-well

171 plates and cultured for $48 \mathrm{~h}$. When $80 \%$ confluency was reached, cells were inoculated with the

172 Mexico ZIKV strain at $400 \mathrm{TCID}_{50} / \mathrm{ml}$ (or an identical volume of the mock culture).

173 Alternatively, $10 \mu \mathrm{g} / \mathrm{ml}$ or $5 \mu \mathrm{g} / \mathrm{ml}$ of the translation inhibitor cycloheximide (CHX; stock

174 solution $100 \mathrm{mg} / \mathrm{ml}$; Sigma-Aldrich) was added to A549 cells and U251 cells, respectively, or

$17510 \mu \mathrm{M}$ eIF4G1 inhibitor (4EGI-1; stock solution $10 \mathrm{mM}$; Biotechne)(32) was added. At $48 \mathrm{~h}$

176 p.i., cells were washed twice with PBS and incubated in unsupplemented DMEM for $2 \mathrm{~h}$ at

$17737^{\circ} \mathrm{C}$. The supernatant was discarded, fresh DMEM medium supplemented with $3 \% \mathrm{FBS}$, and

$1781 \mu \mathrm{M}$ puromycin (stock solution; Merck) was added, and cells were further incubated for 30

$179 \min$ at $37^{\circ} \mathrm{C}$. Subsequently, cells were collected for western blotting analysis.

180

181 SDS-PAGE and western blotting

182 Cells grown in 6-well, 12-well, or 24-well plates were lysed for 30 min on ice in $200 \mu 1$, $183100 \mu \mathrm{l}$, or $50 \mu \mathrm{l}$ RIPA buffer $(50 \mathrm{mM}$ TRIS, $150 \mathrm{mM} \mathrm{NaCl}, 1 \%$ Nonidet P-40, 0.5\% sodium

184 deoxycholate, $0.1 \%$ SDS, pH 7.5), respectively. Subsequently, samples were centrifuged

$185\left(10 \mathrm{~min}\right.$ at $\left.13,000 \times \mathrm{g}, 4^{\circ} \mathrm{C}\right)$, and the pelleted cell debris was discarded. The total protein 186 concentration in each sample supernatant was quantified using the bicinchoninic acid (BCA) 
187 method (Pierce BCA Protein Assay Kit; Thermo Scientific) according to the manufacturer's

188 protocol. Supernatants were mixed 5:1 with denaturing buffer (202.5 mM TRIS pH 6.8, 10\%

189 SDS, $15 \% \beta$-mercaptoethanol, 30\% glycerol, $0.3 \%$ Bromophenol Blue) and boiled at $95^{\circ} \mathrm{C}$ for

$1905 \mathrm{~min}$.

191 For detection of proteins, lysates were loaded and separated on $12 \%$ polyacrylamide gels,

192 and separated by SDS-PAGE over $2 \mathrm{~h}$ at $120 \mathrm{~V}$. BlueStar Plus Prestained Protein Markers

193 (NIPPON Genetics, Germany) were used for reference. Subsequently, gels were subjected to

194 wet electrotransfer onto methanol-activated polyvinylidene difluoride (PVDF; GE Healthcare,

195 Poland) membranes in $25 \mathrm{mM}$ TRIS, $192 \mathrm{mM}$ glycine, 20\% methanol buffer for $1 \mathrm{~h}$ at $100 \mathrm{~V}$.

196 Following transfer, nonspecific binding sites were blocked with 5\% skimmed milk (BioShop,

197 Canada) in TRIS-buffered saline (20 mM TRIS, $0.5 \mathrm{M} \mathrm{NaCl}, \mathrm{pH} 7.5$ ) supplemented with $0.05 \%$

198 Tween 20 (TBS-T) by overnight incubation at $4^{\circ} \mathrm{C}$. To detect NS3 protein, membranes were

199 incubated with a rabbit anti-NS3 antibody (1:1000, GeneTex, USA) followed by a secondary

200 goat anti-rabbit antibody (1:20000; Dako, Denmark) conjugated with horseradish peroxidase

201 (HRP). To detect the eIF4G1 protein, membranes were incubated with a rabbit anti-eIF4G1

202 antibody (1:1000; Thermo Scientific) followed by a secondary goat anti-rabbit antibody

203 (1:20000; Dako) conjugated with HRP. For puromycin detection, membranes were incubated

204 with mouse anti-puromycin antibody (1:10000; Merck) followed by a secondary rabbit anti-

205 mouse antibody (1:20000; Dako) conjugated with HRP. To detect GAPDH protein, membranes

206 were incubated with a rabbit anti-GAPDH antibody (1:5000; Cell Signaling) followed by a

207 secondary goat anti-rabbit antibody (1:20000; Dako) conjugated with HRP. All antibodies were

208 diluted in 1.5\% skimmed milk in TBS-T. The signal was developed using Immobilon Western

209 Chemiluminescent HRP Substrate (Millipore, Poland) and recorded with a ChemiDoc Imaging

210 System (Bio-Rad, Poland). 


\section{Isolation of nucleic acid and reverse transcription}

213 Viral RNA was isolated from $100 \mu \mathrm{l}$ cell culture supernatant using a viral DNA/RNA

214 Isolation Kit (A\&A Biotechnology, Poland) according to the manufacturer's protocol. Reverse

215 transcription was carried out using a High Capacity cDNA Reverse Transcription Kit (Thermo

216 Scientific) according to the manufacturer's protocol. cDNA samples were prepared in $10 \mu 1$

217 volumes using a High Capacity cDNA Reverse Transcription Kit (Thermo Scientific) according

218 to the manufacturer's instructions. The reaction was carried out for $10 \mathrm{~min}$ at $25^{\circ} \mathrm{C}, 120 \mathrm{~min}$ at

$21937^{\circ} \mathrm{C}$, and 5 min at $85^{\circ} \mathrm{C}$.

220

221 Quantitative PCR (qPCR)

222 Zika virus RNA yields were assessed using real-time PCR on a 7500 Fast Real-Time PCR

223 instrument (Thermo Scientific, Poland). ZIKV cDNA was amplified in a reaction mixture

224 containing $1 \times$ TaqMan Universal PCR Master Mix (RT-PCR mix; A\&A) in the presence of 225 FAM/TAMRA (6-carboxyfuorescein/6-carboxytetramethylrhodamine) probe (5' CGG CAT 226 ACA GCA TCA GGT GCA TAG GAG 3'; $100 \mathrm{nM}$ ) and primers (5' TTG GTC ATG ATA 227 CTG CTG ATT GC 3' and 5' CCT TCC ACA AAG TCC CTA TTG C 3'; 450 nM each). The 228 reaction was carried out for $2 \mathrm{~min}$ at $50^{\circ} \mathrm{C}$ and $10 \mathrm{~min}$ at $92^{\circ} \mathrm{C}$, followed by 40 cycles at $92^{\circ} \mathrm{C}$ 229 for $15 \mathrm{~s}$ and $60^{\circ} \mathrm{C}$ for $1 \mathrm{~min}$. DNA standards were subjected to qPCR along with the cDNA. 230 Rox was used as a reference dye. 


\section{RESULTS}

\section{Expression of Zika virus protease in eukaryotic cells}

235 In this study, we expressed and purified full-length NS2B-NS3 protein without linkers. The

236 NS2B-NS3 protein and its inactive Ser135Ala variant were expressed from pBudCE4.1

237 plasmids in $293 \mathrm{~T}$ (left panel) and A549 (right panel) cells. Western blotting with anti-NS3

238 antibody (full-length recombinant Zika virus NS3 protein was used as a positive control)

239 detected NS2B-NS3 ${ }^{\mathrm{WT}}$ and NS2B-NS3 ${ }^{\mathrm{S} 135 \mathrm{~A}}$ in cell lysates prepared from cells collected at $48 \mathrm{~h}$

240 post-transfection. The band corresponding to NS3/NS2B was expected to migrate at $\sim 82 \mathrm{kDa}$,

241 but the active protease should undergo autocatalytic processing to yield the mature $\sim 68 \mathrm{kDa}$

242 NS3 protein (Fig. 1A). Processing should also result in the generation of the smaller $14 \mathrm{kDa}$

243 NS2B (Fig. 1B). All fragments were observed as expected, confirming the activity of the

244 protease, and protein expression was efficient in both cell lines.

245

246

247

248

249

250

251

252

253

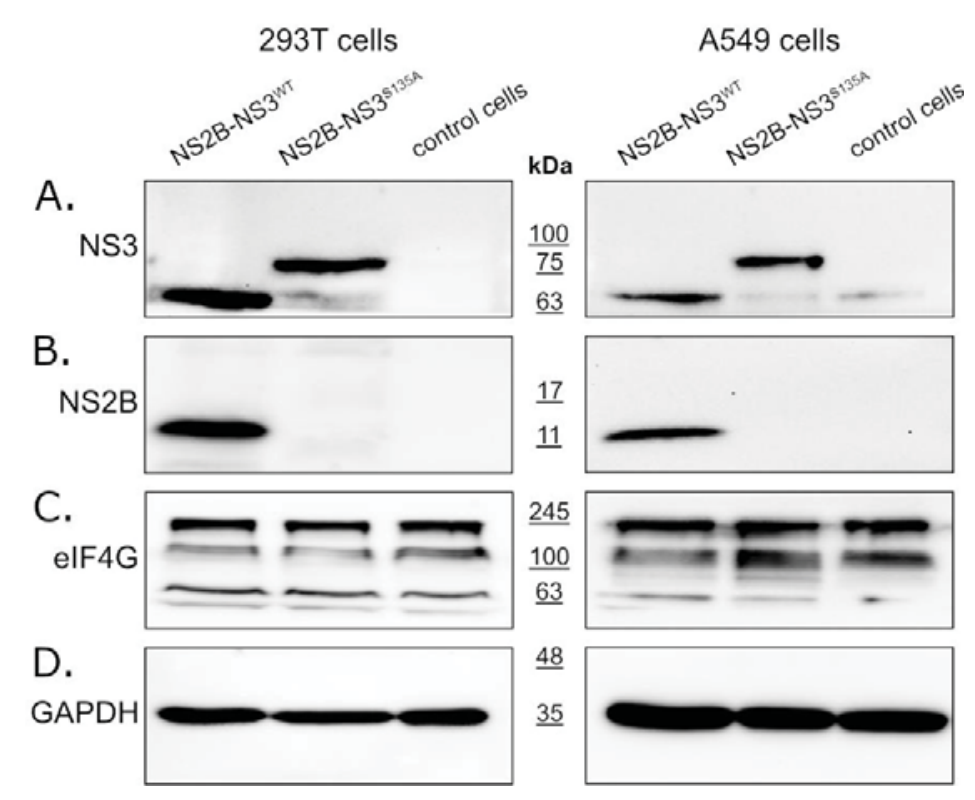

Fig. 1 Expression of active and inactive NS2B-NS3 protease from Zika virus in eukaryotic cells does not affect elF4G1 protein levels. 293T (left panel) and A549 (right) cells expressing NS2B-NS3 ${ }^{\text {WT }}$ or NS2B$\mathrm{NS}^{\mathrm{S}}{ }^{135 \mathrm{~A}}$ were assessed alongside control cells. Cells were harvested at $48 \mathrm{~h}$ after transfection, lysed, and virus lysates were analyzed by western blotting. Virus infection was confirmed by the presence of NS3 (A) and NS2B (B) proteins. Anti-elF4G1 antibody was used to detect and compare changes in elF4G1 protein abundance in cells expressing active or inactive protease, relative to control cells (C). The GAPDH protein was used as a reference to ensure that identical amounts of proteins were present in each sample (D). 


\section{$255 \quad$ NS3 protease does not affect eIF4G1 levels}

256 To verify whether the ZIKV NS3 protease has any effect on eIF4G1 levels, cells expressing

257 either active or inactive protease were analyzed used western blotting, alongside control cells

258 lacking the protease. The results (Fig. 1C) showed that eIF4G1 migrated at $\sim 188 \mathrm{kDa}$, and

259 isoforms with lower molecular masses were also visible. While we observed high variability in

260 eIF4G1 content depending on the culture time, temperature, and general cell conditions, there

261 were no differences in protein abundance in cells expressing active or inactive protease, or

262 control cells (data not shown), and this was the case for both 293T and A549 cells.

263

264 Zika virus infection does not result in altered eIF4G1 levels

265 Since eIF4G1 levels were not affected by the expression of NS3 protease, we assessed

266 whether the eIF4G1 protein is cleaved or degraded during virus infection using ZIKV-infected

267 and mock-infected $293 \mathrm{~T}$ and A549 cells. First, we confirmed virus replication in cells through

268 NS3 and NS2B protein expression using western blotting (Fig. 2A, B). Levels of the eIF4G1

269 protein were then assessed in virus-infected and mock-inoculated cells, but there were no

270 differences in eIF4G1 protein abundance. 
271

272

273

274

275

276

277

278

279

280

281

282

283

284

285

286

287

288

289

290

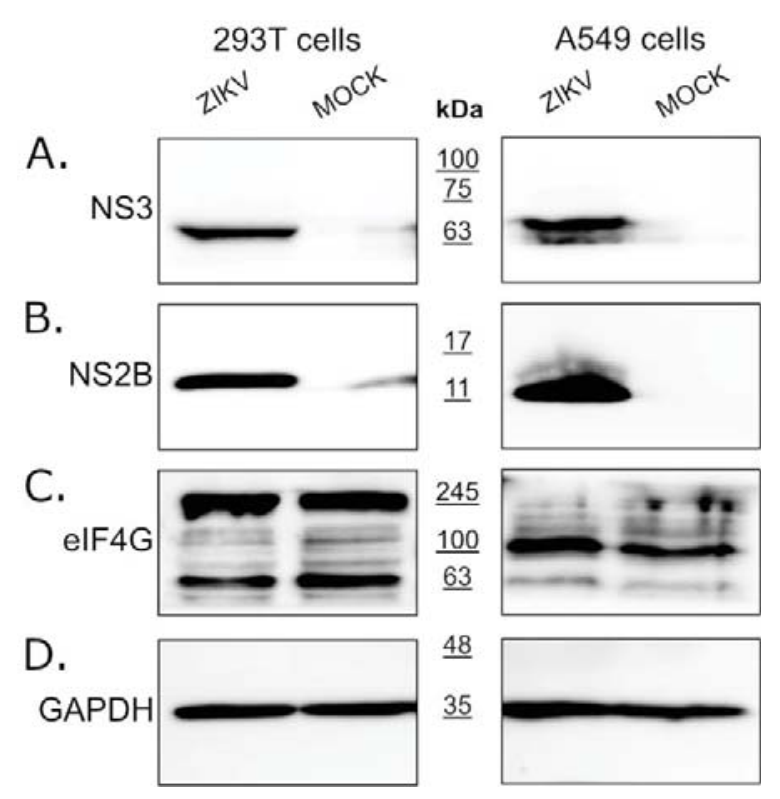

Fig. 2 Expression of the elF4G1 protein in ZIKV-infected and mock-infected cells. 293T (left panel) and A549 (right panel) cells were infected with ZIKV, or inoculated with mock or virus lysates, and analyzed by western blotting. Virus infection was confirmed by the presence of NS3 (A) and NS2B (B) proteins. The elF4G1 protein was detected using anti-elF4G1 antibody (C). The GAPDH protein was used as a reference to ensure that identical amounts of proteins were present in each sample (D).

\section{ZIKV does not hamper production of cellular proteins by altering levels of transcription factors}

It was suggested that ZIKV NS3 cleaves eIF4G1 to redirect the cellular machinery toward viral protein production, which may be independent of cellular transcription factors(33). To test this hypothesis, the host protein synthesis efficiency was evaluated using surface sensing of translation (SUnSET) assays to measure protein synthesis in cultured cells (34). Puromycin can mimic the aminoacyl end of aminoacyl-tRNAs, and it is partially incorporated in synthesized proteins. The incorporation rate reflects the rate of mRNA translation. Puromycin incorporation was detected by western blotting using anti-puromycin antibodies. Two reference inhibitors were also tested: the protein synthesis inhibitor cycloheximide (CHX) and the eIF4G1-specific inhibitor 4EGI1 (Fig. 3A). In samples treated with either CHX or 4EGI1, the synthesis of proteins was significantly hampered, but protein synthesis in ZIKV-infected cells was not altered. The cytotoxicity of the inhibitors was also evaluated (Fig. 3B). 
A

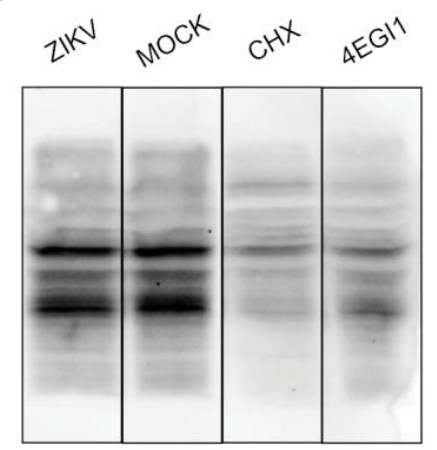

B

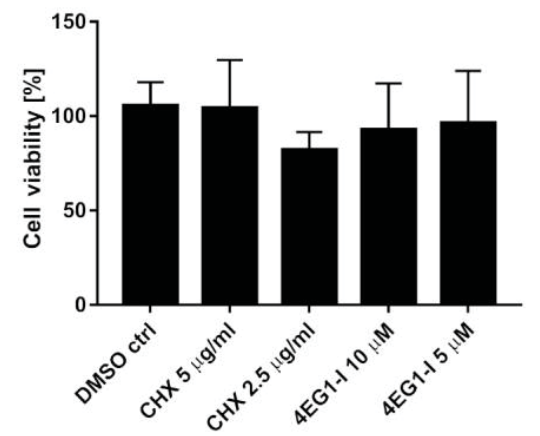

\section{1}

292

293

294

295

296

297

\section{8}

299

300

301

302

303

304

305

306

307 308

Fig. 3 ZIKV infection does not inhibit the translation of host proteins. SUnSet assays were performed on ZIKV- and mock-infected A549 cells, and cells treated with cycloheximide or 4EGI1 inhibitors. The presence of puromycin protein was detected using anti-puromycin antibody (A). Cell viability was evaluated relative to control cells treated with DMSO alone. The assay was performed in triplicate, and average values with standard errors are presented (B).

\section{Overexpression of eIF4G1 does not limit ZIKV replication}

To verify whether eIF4G1 expression negatively regulates replication of ZIKV, 293T cells were transfected with a plasmid encoding eIF4G1 (or GFP as a control). Protein levels were verified using western blotting (Fig. 4A), and ZIKV replication was evaluated in nontransfected cells, GFP-expressing cells, and eIF4G-expressing cells. Different strains of Zika virus were used to ensure that any effect is not limited to a single lineage. Cells were infected, and at a single timepoint cell culture supernatants were collected for RNA isolation and subsequent RT-qPCR assessment of the virus yield. Although virus yields varied depending on the virus strain, no inhibition of virus replication was observed for any of the tested strains.

These results show that NS3-mediated loss of function of the eIF4G1 protein was not beneficial for virus replication (Fig. 4B). 
A

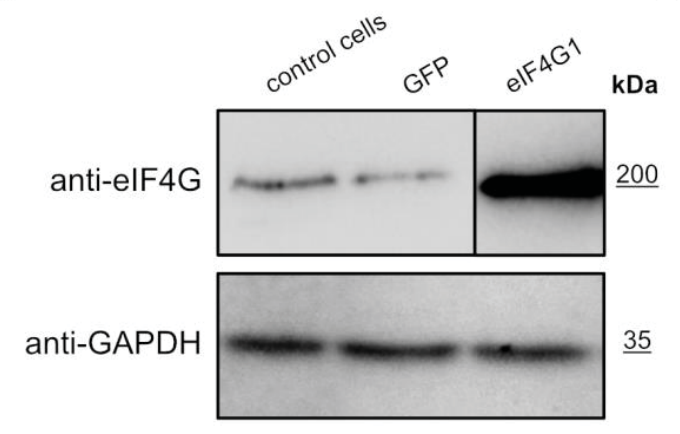

309

310

311

312

313

314

315

316

317

318

319

320

321

322

323

324

325

326 average values with standard errors are presented (B).

\section{eIF4G supports replication of ZIKV}

B

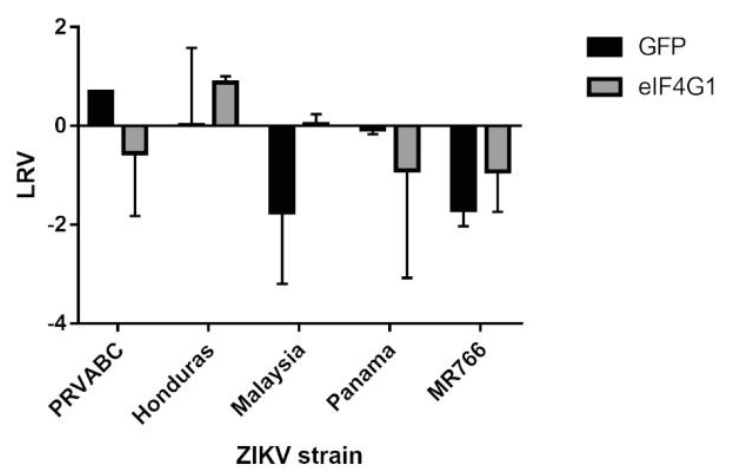

Fig. 4 ZIKV replication in elF4G1-overexpressing cells. Western blotting analysis (anti-elF4G1 antibodies) was performed on 293T cells transfected with plasmid encoding elF4G1 or GFP. The GAPDH protein was used as a reference to ensure that identical amounts of proteins were present in each sample (A). ZIKV virus replication in 293 T cells transfected with plasmid encoding elF4G1 or GFP. The virus yield was assessed by RT-qPCR. The y-axis represents the log reduction value (LRV) in virus yield in treated samples, and the $\mathrm{x}$-axis corresponds to different ZIKV strains. The assay was performed in triplicate, and

To further investigate the role of eIF4G1, we silenced its expression in 293T cells and probed ZIKV replication in these cells. Briefly, cultures were transfected with eIF4G1 siRNA or with scrambled siRNA. Silencing was confirmed by western blotting using antibodies specific to eIF4G1 (Fig. 5A). Cells were infected with ZIKV and incubated for 3 days at $37^{\circ} \mathrm{C}$, after which culture supernatants were collected, RNA was isolated and reverse transcribed, and virus replication was evaluated by $\mathrm{qPCR}$. Again, we did not observe an increase in virus production; on the contrary, for some strains, silencing led to inhibition of virus replication relative to control cells or cells transfected with scrambled siRNA (Fig. 5B). 
A

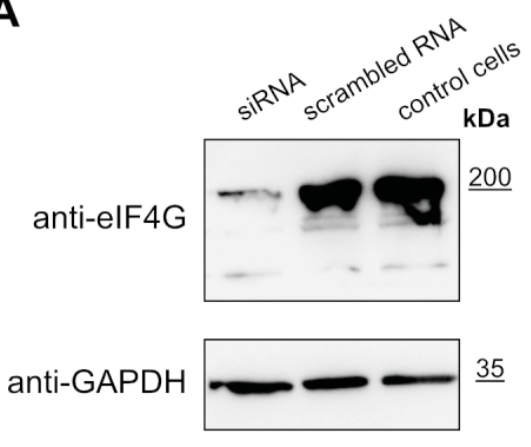

327

328

329

330

331

332

333

334

335

336

337

338

339

340

341

342

343

344 are presented (B).

\section{Discussion}

\section{Table 1.}

B

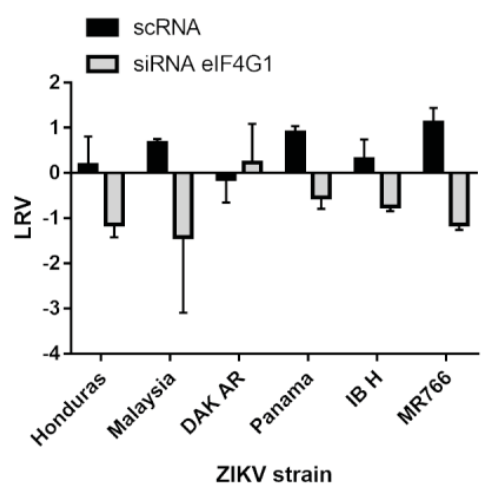

Fig. 5 ZIKV replication in cells lacking elF4G1. A549 cells were transfected with elF4G1 siRNA or scrambled siRNA. Non-transfected controls were included. The GAPDH protein was used as a reference to ensure that identical amounts of proteins were present in each sample (A). ZIKV virus replication in A549 cells transfected with different siRNAs. The virus yield was assessed by RT-qPCR. The y-axis represents the log reduction value (LRV) in virus yield in treated samples, and the $\mathrm{x}$-axis corresponds to different ZIKV strains. The assay was performed in triplicate, and average values with standard errors

This study aimed to verify the role of the ZIKV NS3 protein in the remodeling of host cells. NS3 protease is essential for virus replication because it is required for viral protein maturation (35-37). However, viral proteases are generally considered to be highly specific enzymes that co-evolved with the host, and they typically target specific cellular pathways to support viral replication in the host cell, or to block recognition of the virus by the host immune system (38, 39). In the case of ZIKV, some protease targets have been identified, and these are listed in 
Table 1. Reported NS3 protease targets in the cell.

\begin{tabular}{|c|c|c|c|c|c|c|}
\hline & PROTEIN & FUNCTION & PROTEASE EXPRESSION & CELLULAR MODEL & IDENTIFICATION & REFERENCES \\
\hline 1 & $\begin{array}{l}\text { autophagy-related } \\
\text { protein } \quad 16-1 \\
(\text { ATG16L1) }\end{array}$ & Autophagy & $\begin{array}{l}\text { expression in the prokaryotic system; construct } \\
\text { based on } 48-100 \text { aa residues of NS2B and 1-178 } \\
\text { aa of NS3 (protease domain) }\end{array}$ & $\begin{array}{l}\text { protease substrate verification in } \\
\text { the cellular lysate of } 293 \mathrm{~T} \text { and } \\
\text { A549 cells treated with purified } \\
\text { protease; }\end{array}$ & $\begin{array}{l}\text { mass spectrometry; Western } \\
\text { blot analysis }\end{array}$ & $(33)$ \\
\hline 2 & $\begin{array}{l}\text { eukaryotic } \\
\text { translation initiation } \\
\text { factor } 4 \text { gamma } \\
(\mathrm{eIF} 4 \mathrm{G} 1)\end{array}$ & $\begin{array}{l}\text { Translation } \\
\text { process }\end{array}$ & $\begin{array}{l}\text { expression in prokaryotic system; construct based } \\
\text { on } 48-100 \text { aa residues of NS2B and } 1-178 \text { aa of } \\
\text { NS3 (protease domain) }\end{array}$ & $\begin{array}{l}\text { protease substrate verification in } \\
\text { cellular lysate of } 293 \mathrm{~T} \text { and A549 } \\
\text { cells treated with purified protease; }\end{array}$ & $\begin{array}{l}\text { mass spectrometry; Western } \\
\text { blot analysis }\end{array}$ & $(33)$ \\
\hline 3 & FAM134B & Reticulofagy & $\begin{array}{l}\text { expression in eukaryotic cells; the NS2B-NS3 } \\
\text { protein coding region was amplified from cDNA } \\
\text { produced from HBMEC infected with ZIKV } \\
\text { MR766; cells transfection }\end{array}$ & $\begin{array}{l}\text { HBMEC, U2OS, 293T and HeLa } \\
\text { cells }\end{array}$ & $\begin{array}{l}\text { Western blots and the } \\
\text { fluorescence microscopy } \\
\text { analysis }\end{array}$ & $(40)$ \\
\hline 4 & Septin-2 & Cytokinesis & $\begin{array}{l}\text { expression in eukaryotic cells, cells transfection, } \\
\text { and transduction with lentiviral vectors; }\end{array}$ & $\begin{array}{l}\text { HeLa and } 293 \mathrm{~T} \text { cells and human } \\
\text { neural progenitor cells }\end{array}$ & $\begin{array}{l}\text { mass spectrometry; Western } \\
\text { blots and fluorescence } \\
\text { microscopy analysis, pull- } \\
\text { down assay }\end{array}$ & $(41)$ \\
\hline 5 & $\begin{array}{l}\text { disulfide-isomerase } \\
\text { A3 (PDIA3) }\end{array}$ & $\begin{array}{l}\text { ER stress } \\
\text { response }\end{array}$ & $\begin{array}{l}\text { Expression in eukaryotic cells, cells transfection; } \\
\text { construct based on 48-94 aa residues of NS2B and } \\
1-188 \text { aa of NS3 (protease domain) }\end{array}$ & 293T and A549 cells & $\begin{array}{l}\text { mass spectrometry and } \\
\text { Western blot analysis }\end{array}$ & $(42)$ \\
\hline 6 & $\begin{array}{l}\text { aldolase } \\
\text { (ALDOA) }\end{array}$ & glycolysis & $\begin{array}{l}\text { Expression in eukaryotic cells, cells transfection; } \\
\text { construct based on 48-94 aa residues of NS2B and } \\
1-188 \text { aa of NS3 (protease domain) }\end{array}$ & 293T and A549 cells & $\begin{array}{l}\text { mass } \quad \text { spectrometry and } \\
\text { Western blot analysis }\end{array}$ & $(42)$ \\
\hline 7 & $\begin{array}{l}\text { Nup98, Nup153 and } \\
\text { TPR }\end{array}$ & \begin{tabular}{|lr} 
Formation & of \\
nuclear pore \\
complex
\end{tabular} & Expression in eukaryotic cells, cells transfection & Huh-7 cells & $\begin{array}{l}\text { Western blots and the } \\
\text { fluorescence microscopy } \\
\text { analysis }\end{array}$ & $(43)$ \\
\hline
\end{tabular}


In the present work, we reviewed the published data and verified these potential protease targets experimentally by measuring changes in the levels of potential cellular targets in the presence of active or inactive protease. Since in some cases the localization or specificity of the protease may differ in the absence of other viral proteins, we also measured the levels of potential NS3 targets in ZIKV-infected cells.

We employed eIF4G1 as a model protease substrate because we believe that processing of

354 this protein has straightforward consequences for both host cells and virus. The eIF4G protein

355 is involved in the translation process by serving as a eukaryotic translation initiation factor.

356 Together with eIF4A and eIF4E, eIF4G forms the EIF4F multi-subunit protein complex, which 357 recognizes the mRNA cap and facilitates the recruitment of mRNA to the ribosome. eIF4G 358 serves mainly as a linker that forms a scaffold for the complex (44). Interestingly, some viruses 359 are reported to target this protein, and thereby rewire the cellular machinery and switch off 360 cellular protein production. For example, coxsackievirus B3 virus-encoded protease cleaves 361 eIF4G1, but the resulting suppression of cellular translation does not affect viral replication,

362 since picornaviruses utilize the IRES rather than cap-dependent translation initiation (25-27).

363 Consequently, the complete protein production machinery serves viral replication. Similarly,

364 for some flaviviruses, it was postulated that the $5^{\prime}$ '-untranslated region (5'-UTR) may act as an

365 IRES, and NS3 protease encoded in the flaviviral genome may target cellular translation 366 initiation factors (45).

367 Herein, we first tested whether overexpression of NS2B/NS3 had any effect on levels of 368 the eIF4G1 protein, as reported previously by Hill et al. (2018). Notably, the authors of this 369 work performed their analysis using ZIKV protease expressed in a prokaryotic system, which 370 was purified and mixed with cellular lysates from 293 T and A549 cells (33). In our current 371 work, we expressed part of the ZIKV genome encompassing the NS2B and NS3 proteins. Our 372 approach allowed us to anchor the NS3 protease in the ER membrane via the NS2B cofactor. 
373 Furthermore, using this approach, we were able to monitor whether the protease was active in

374 every experiment because it was autocatalytically (in trans and cis) processing its natural 375 substrate (the NS2B/NS3 junction). The catalytically inactive mutant was used as a negative 376 control. Protein content analysis did not reveal any significant decrease in eIF4G1 protein 377 levels. However, the experimental setup used in the present study may not be entirely 378 appropriate, since it may not accurately recapitulate protease activity and localization during 379 natural viral infection. To ensure that the observed effect was not an artifact, cells were infected 380 with ZIKV, and eIF4G1 levels were measured. Because it remains disputable whether a specific 381 decrease in the level of a particular protein is reflected by changes in signal transduction, we 382 tested the effect of ZIKV infection on the production of cellular proteins using the puromycin 383 assay, with appropriate controls (32)(46). We did not observe any changes in host gene 384 translation, proving that the effect on eIF4G1 cleavage is not likely to alter the physiology of 385 the cell. However, modulation may occur locally at the replication site, and while it would 386 improve viral replication, the effect on the whole cell may be too subtle to be detected. For this 387 reason, the effect of eIF4G1 on ZIKV replication was tested by gene silencing and gene 388 overexpression experiments, but the role of the eIF4G1 protein in viral replication remained 389 elusive.

390 As listed in Table 1, several proteins have been reported as targets for the ZIKV NS3 391 protease. Intrigued by the results obtained for the eIF4G1 protein, we explored whether 392 autophagy-related protein 16-1 (ATG16L1), c-Jun amino-terminal kinase-interacting protein 4 393 (JIP4), mitogen-activated protein kinase kinase kinase 7 (TAK1 or MAP3K7), disulfide394 isomerase A3 (PDIA3), heterogeneous nuclear ribonucleoprotein A2/B1 (hnRNP A2/B1), 395 aldolase A (ALDOA) (42), ER-localized reticulophagy receptor FAM134B (40), and septin-2 396 protein (41) may serve as targets for the NS3 protease. To our surprise, we could not confirm 397 these previous observations, and we considered why this might be the case. First, in these 
398 previous studies, different expression systems and constructs were employed. In some cases,

399 part of the NS2B cofactor was covalently linked to NS3 by a flexible linker. This is relevant, 400 as it has been shown by others that the linker itself may alter the dynamics of the protein and,

401 consequently, the substrate specificity of the protease. Second, the soluble version of the

402 protease is not anchored at the membrane, which may also alter the substrate specificity. Third,

403 the localization of the protease in the ER may limit the number of possible targets, and even

404 proteins that may serve as NS3 substrates in biochemical assays may not be cleaved due to the

405 differential spatial distribution. Finally, although mass spectrometry is sensitive enough to

406 detect even minor changes in protein content, it could in some cases deliver results which are

407 not relevant for the homeostasis of the intracellular environment. Therefore proteome changes

408 detected by MS should be confirmed by other methods, and their biological relevance should

409 be explored, using other approaches.

410 In conclusion, our study shows that the biological role of the ZIKV NS3 protease may be

411 limited. This may be due to the relatively recent transmission of the virus on a large scale to the

412 human population. It would therefore be interesting to analyze virus evolution in humans in the

413 future, especially changes in the localization and/or substrate specificity of NS3 protease.

414

\section{$415 \quad$ Funding}

416 This work was supported by the National Science Centre, Poland, in the form of Grant No.

417 2016/21/B/NZ6/01307 to K.P and P.S. The funders had no role in study design, data collection

418 and analysis, decision to publish, or preparation of the manuscript.

\section{References}


423 1. Dick, G. W., and Haddow, A. J. (1952) Uganda S virus; a hitherto unrecorded virus 424 isolated from mosquitoes in Uganda. I. Isolation and pathogenicity. Trans $R$ Soc Trop Med 425 Hyg 46, 600-618

426 2. Duffy, M. R., Chen, T. H., Hancock, W. T., Powers, A. M., Kool, J. L., Lanciotti, R. S., 427 Pretrick, M., Marfel, M., Holzbauer, S., Dubray, C., Guillaumot, L., Griggs, A., Bel, M., 428 Lambert, A. J., Laven, J., Kosoy, O., Panella, A., Biggerstaff, B. J., Fischer, M., and Hayes, E. B. (2009) Zika virus outbreak on Yap Island, Federated States of Micronesia. N Engl J Med 360, 2536-2543

3. Musso, D., Ko, A. I., and Baud, D. (2019) Zika Virus Infection - After the Pandemic. N Engl J Med 381, 1444-1457

4. Campos, G. S., Bandeira, A. C., and Sardi, S. I. (2015) Zika Virus Outbreak, Bahia, Brazil. Emerg Infect Dis 21, 1885-1886

5. Uncini, A., Shahrizaila, N., and Kuwabara, S. (2017) Zika virus infection and GuillainBarré syndrome: a review focused on clinical and electrophysiological subtypes. J Neurol Neurosurg Psychiatry 88, 266-271

6. Barbi, L., Coelho, A. V. C., Alencar, L. C. A., and Crovella, S. (2018) Prevalence of Guillain-Barré syndrome among Zika virus infected cases: a systematic review and metaanalysis. Braz J Infect Dis 22, 137-141

7. Nascimento, O. J. M., and da Silva, I. R. F. (2017) Guillain-Barré syndrome and Zika virus outbreaks. Curr Opin Neurol 30, 500-507

8. Vissoci, J. R. N., Rocha, T. A. H., Silva, N. C. D., de Sousa Queiroz, R. C., Thomaz, E. B. A. F., Amaral, P. V. M., Lein, A., Branco, M. D. R. F., Aquino, J., Rodrigues, Z. M. R., da Silva, A. A. M., and Staton, C. (2018) Zika virus infection and microcephaly: Evidence regarding geospatial associations. PLoS Negl Trop Dis 12, e0006392

9. Barreto, M. L., Barral-Netto, M., Stabeli, R., Almeida-Filho, N., Vasconcelos, P. F. C., Teixeira, M., Buss, P., and Gadelha, P. E. (2016) Zika virus and microcephaly in Brazil: a scientific agenda. Lancet 387, 919-921

450 10. Mlakar, J., Korva, M., Tul, N., Popović, M., Poljšak-Prijatelj, M., Mraz, J., Kolenc, M., 451 Resman Rus, K., Vesnaver Vipotnik, T., Fabjan Vodušek, V., Vizjak, A., Pižem, J., Petrovec, 452 M., and Avšič Županc, T. (2016) Zika Virus Associated with Microcephaly. N Engl J Med 374, 951-958

11. Fields, B. N., Knipe, D. M., and Howley, P. M. (2013) Fields virology, 6th Ed., Wolters Kluwer Health/Lippincott Williams \& Wilkins, Philadelphia

12. Hoffmann, H. H., Schneider, W. M., Blomen, V. A., Scull, M. A., Hovnanian, A., Brummelkamp, T. R., and Rice, C. M. (2017) Diverse Viruses Require the Calcium Transporter SPCA1 for Maturation and Spread. Cell Host Microbe 22, 460-470 e465

13. Bessaud, M., Pastorino, B. A., Peyrefitte, C. N., Rolland, D., Grandadam, M., and Tolou, H. J. (2006) Functional characterization of the NS2B/NS3 protease complex from seven viruses belonging to different groups inside the genus Flavivirus. Virus Res 120, 79-90

14. Chambers, T. J., Grakoui, A., and Rice, C. M. (1991) Processing of the yellow fever virus nonstructural polyprotein: a catalytically active NS3 proteinase domain and NS2B are required for cleavages at dibasic sites. $J$ Virol 65, 6042-6050

15. Falgout, B., Miller, R. H., and Lai, C. J. (1993) Deletion analysis of dengue virus type 4 nonstructural protein NS2B: identification of a domain required for NS2B-NS3 protease activity. $J$ Virol 67, 2034-2042

16. Lescar, J., Luo, D., Xu, T., Sampath, A., Lim, S. P., Canard, B., and Vasudevan, S. G. (2008) Towards the design of antiviral inhibitors against flaviviruses: the case for the multifunctional NS3 protein from Dengue virus as a target. Antiviral Res 80, 94-101

17. Leung, D., Schroder, K., White, H., Fang, N. X., Stoermer, M. J., Abbenante, G., Martin, J. L., Young, P. R., and Fairlie, D. P. (2001) Activity of recombinant dengue 2 virus NS3 
473 protease in the presence of a truncated NS2B co-factor, small peptide substrates, and inhibitors.

474 J Biol Chem 276, 45762-45771

475 18. Aguilera-Pesantes, D., and Méndez, M. A. (2017) Structure and sequence based 476 functional annotation of Zika virus NS2b protein: Computational insights. Biochem Biophys 477 Res Commun 492, 659-667

478 19. Erbel, P., Schiering, N., D'Arcy, A., Renatus, M., Kroemer, M., Lim, S. P., Yin, Z., 479 Keller, T. H., Vasudevan, S. G., and Hommel, U. (2006) Structural basis for the activation of 480 flaviviral NS3 proteases from dengue and West Nile virus. Nat Struct Mol Biol 13, 372-373

481 20. Xing, H., Xu, S., Jia, F., Yang, Y., Xu, C., Qin, C., and Shi, L. (2020) Zika NS2B is a 482 crucial factor recruiting NS3 to the ER and activating its protease activity. Virus Res 275, 483197793

484 21. Chatel-Chaix, L., Germain, M. A., Götte, M., and Lamarre, D. (2012) Direct-acting and 485 host-targeting HCV inhibitors: current and future directions. Curr Opin Virol 2, 588-598

486 22. Chatel-Chaix, L., Baril, M., and Lamarre, D. (2010) Hepatitis C Virus NS3/4A Protease 487 Inhibitors: A Light at the End of the Tunnel. Viruses 2, 1752-1765

488 23. McCauley, J. A., and Rudd, M. T. (2016) Hepatitis C virus NS3/4a protease inhibitors. 489 Curr Opin Pharmacol 30, 84-92

490 24. Croft, S. N., Walker, E. J., and Ghildyal, R. (2018) Human Rhinovirus 3C protease 491 cleaves RIPK1, concurrent with caspase 8 activation. Sci Rep 8, 1569

492 25. Avanzino, B. C., Fuchs, G., and Fraser, C. S. (2017) Cellular cap-binding protein, 493 eIF4E, promotes picornavirus genome restructuring and translation. Proc Natl Acad Sci U S A 494 114, 9611-9616

495 26. Chase, A. J., and Semler, B. L. (2012) Viral subversion of host functions for 496 picornavirus translation and RNA replication. Future Virol 7, 179-191

497 27. Glaser, W., and Skern, T. (2000) Extremely efficient cleavage of eIF4G by picornaviral 498 proteinases L and 2A in vitro. FEBS Lett 480, 151-155

499 28. Li, K., Foy, E., Ferreon, J. C., Nakamura, M., Ferreon, A. C., Ikeda, M., Ray, S. C., 500 Gale, M., and Lemon, S. M. (2005) Immune evasion by hepatitis C virus NS3/4A protease501 mediated cleavage of the Toll-like receptor 3 adaptor protein TRIF. Proc Natl Acad Sci U S A $502 \quad 102,2992-2997$

503 29. Meylan, E., Curran, J., Hofmann, K., Moradpour, D., Binder, M., Bartenschlager, R., 504 and Tschopp, J. (2005) Cardif is an adaptor protein in the RIG-I antiviral pathway and is 505 targeted by hepatitis C virus. Nature 437, 1167-1172

506 30. Anggakusuma, Brown, R. J. P., Banda, D. H., Todt, D., Vieyres, G., Steinmann, E., and 507 Pietschmann, T. (2016) Hepacivirus NS3/4A Proteases Interfere with MAVS Signaling in both 508 Their Cognate Animal Hosts and Humans: Implications for Zoonotic Transmission. J Virol 90, 509 10670-10681

510 31. REED, L. J., and MUENCH, H. (1938) A SIMPLE METHOD OF ESTIMATING 511 FIFTY PER CENT ENDPOINTS12. American Journal of Epidemiology 27, 493-497

512 32. Moerke, N. J., Aktas, H., Chen, H., Cantel, S., Reibarkh, M. Y., Fahmy, A., Gross, J. 513 D., Degterev, A., Yuan, J., Chorev, M., Halperin, J. A., and Wagner, G. (2007) Small-molecule 514 inhibition of the interaction between the translation initiation factors eIF4E and eIF4G. Cell $515128,257-267$

516 33. Hill, M. E., Kumar, A., Wells, J. A., Hobman, T. C., Julien, O., and Hardy, J. A. (2018) 517 The Unique Cofactor Region of Zika Virus NS2B-NS3 Protease Facilitates Cleavage of Key 518 Host Proteins. ACS Chem Biol 13, 2398-2405

519 34. Schmidt, E. K., Clavarino, G., Ceppi, M., and Pierre, P. (2009) SUnSET, a 520 nonradioactive method to monitor protein synthesis. Nat Methods 6, 275-277 
521 35. Falgout, B., Pethel, M., Zhang, Y. M., and Lai, C. J. (1991) Both nonstructural proteins 522 NS2B and NS3 are required for the proteolytic processing of dengue virus nonstructural 523 proteins. $J$ Virol 65, 2467-2475

524 36. Liu, W. J., Sedlak, P. L., Kondratieva, N., and Khromykh, A. A. (2002)

525 Complementation analysis of the flavivirus Kunjin NS3 and NS5 proteins defines the minimal 526 regions essential for formation of a replication complex and shows a requirement of NS3 in cis 527 for virus assembly. J Virol 76, 10766-10775

528 37. Gebhard, L. G., Iglesias, N. G., Byk, L. A., Filomatori, C. V., De Maio, F. A., and 529 Gamarnik, A. V. (2016) A Proline-Rich N-Terminal Region of the Dengue Virus NS3 Is Crucial 530 for Infectious Particle Production. J Virol 90, 5451-5461

531 38. Castelló, A., Franco, D., Moral-López, P., Berlanga, J. J., Alvarez, E., Wimmer, E., and 532 Carrasco, L. (2009) HIV- 1 protease inhibits Cap- and poly(A)-dependent translation upon 533 eIF4GI and PABP cleavage. PLoS One 4, e 7997

534 39. Alvarez, E., Menéndez-Arias, L., and Carrasco, L. (2003) The eukaryotic translation 535 initiation factor 4GI is cleaved by different retroviral proteases. $J$ Virol 77, 12392-12400

536 40. Lennemann, N. J., and Coyne, C. B. (2017) Dengue and Zika viruses subvert 537 reticulophagy by NS2B3-mediated cleavage of FAM134B. Autophagy 13, 322-332

538 41. Li, H., Saucedo-Cuevas, L., Yuan, L., Ross, D., Johansen, A., Sands, D., Stanley, V., 539 Guemez-Gamboa, A., Gregor, A., Evans, T., Chen, S., Tan, L., Molina, H., Sheets, N., 540 Shiryaev, S. A., Terskikh, A. V., Gladfelter, A. S., Shresta, S., Xu, Z., and Gleeson, J. G. (2019) 541 Zika Virus Protease Cleavage of Host Protein Septin-2 Mediates Mitotic Defects in Neural 542 Progenitors. Neuron 101, 1089-1098.e1084

543 42. Tangsongcharoen, C., Roytrakul, S., and Smith, D. R. (2019) Analysis of cellular 544 proteome changes in response to ZIKV NS2B-NS3 protease expression. Biochim Biophys Acta 545 Proteins Proteom 1867, 89-97

546 43. De Jesús-González, L. A., Cervantes-Salazar, M., Reyes-Ruiz, J. M., Osuna-Ramos, J. 547 F., Farfán-Morales, C. N., Palacios-Rápalo, S. N., Pérez-Olais, J. H., Cordero-Rivera, C. D., 548 Hurtado-Monzón, A. M., Ruíz-Jiménez, F., Gutiérrez-Escolano, A. L., and Del Ángel, R. M. 549 (2020) The Nuclear Pore Complex: A Target for NS3 Protease of Dengue and Zika Viruses. $550 \quad$ Viruses 12

551 44. Jackson, R. J., Hellen, C. U., and Pestova, T. V. (2010) The mechanism of eukaryotic 552 translation initiation and principles of its regulation. Nat Rev Mol Cell Biol 11, 113-127

553 45. Song, Y., Mugavero, J., Stauft, C. B., and Wimmer, E. (2019) Dengue and Zika Virus 554 5' Untranslated Regions Harbor Internal Ribosomal Entry Site Functions. MBio 10

555 46. Schneider-Poetsch, T., Ju, J., Eyler, D. E., Dang, Y., Bhat, S., Merrick, W. C., Green, 556 R., Shen, B., and Liu, J. O. (2010) Inhibition of eukaryotic translation elongation by 557 cycloheximide and lactimidomycin. Nat Chem Biol 6, 209-217 